\title{
Electronic properties of zigzag silicene nanoribbons with single vacancy defect
}

\author{
Mu Wen Chuan, Kien Liong Wong, Afiq Hamzah, Nurul Ezaila Alias, \\ Cheng Siong Lim, Michael Loong Peng Tan \\ School of Electrical Engineering, Faculty of Engineering, Universiti Teknologi Malaysia, Malaysia
}

\begin{tabular}{|c|c|}
\hline Article Info & ABSTRACT \\
\hline Article history: & Silicene is envisaged as one of the two-dimensional (2D) materials for future \\
\hline Received Dec 3, 2019 & $\begin{array}{l}\text { nanoelectronic applications. In addition to its extraordinary electronic } \\
\text { properties, it is predicted to be compatible with the silicon ( } \mathrm{Si} \text { ) fabrication }\end{array}$ \\
\hline Revised Jan 27, 2020 & technology. By using nearest neighbour tight-binding (NNTB) approach, \\
\hline Accepted Feb 8, 2020 & $\begin{array}{l}\text { the electronic properties of zigzag silicene nanoribbons (ZSiNRs) with single } \\
\text { vacancy }(\mathrm{SV}) \text { defects are modelled and simulated. For } 4-\mathrm{ZSiNR} \text { with } \mathrm{L}=2 \text {, }\end{array}$ \\
\hline Keywords: & $\begin{array}{l}\text { the band structures and density of states (DUS) are computed based on SV } \\
\text { incorporated ZSiNRs at varying defect locations. The results show that }\end{array}$ \\
\hline 2D material & the SV defect will shift the band structure and increase the peak of DOS \\
\hline Nanoelectronics & $\begin{array}{l}\text { while the bandgap remain zero. Inis work provides a theoretical framework } \\
\text { to understand the impact of SV defect which is an inevitable non-ideal effect }\end{array}$ \\
\hline Silicene nanoribbons & during the fabrication of silicene nanoribbons (SiNRs). \\
\hline
\end{tabular}

Vacancy defect All rights reserved.

\section{Corresponding Author:}

Michael Loong Peng Tan,

School of Electrical Engineering, Faculty of Engineering,

Universiti Teknologi Malaysia,

81310 Skudai, Johor, Malaysia.

Email: michael@utm.my

\section{INTRODUCTION}

The success of mechanically exfoliated graphene has driven the rigorous explorations of two-dimensional (2D) materials [1-3]. Silicene is the silicon ( $\mathrm{Si}$ ) counterpart of graphene-like 2D materials which has recently become an interesting research topic $[4,5]$. Besides sharing similar Dirac cone properties [6-8] with graphene, silicene has the potential to be compatible with the semiconductor fabrication processes which depend primarily on Si technology [9]. In 2015, silicene field-effect transistor (FET) was fabricated using grow-transfer-fabrication technique [10], but the electronic properties are greatly degraded by the silver substrate. Hence, it is important to perform computational study on silicene while fabrication researchers are searching for other breakthroughs in silicene fabrication technique.

Vacancy defect is an unpreventable non-ideal effect during fabrication process which can significantly affect the electronic properties of the fabricated material $[11,12]$. Although defects are undesirable events, they can sometimes enhance the electronic properties of the material for specific application [13]. In this paper, the effects of single vacancy (SV) defect on the electronic properties of zigzag silicene nanoribbons (ZSiNRs) were modelled using nearest neighbour tight-binding (NNTB). Figure 1 illustrates the schematic atomic arrangedment of a ZSiNR. This work has considered only zigzag edge because ZSiNRs possess semi-metallic properties for all nanoribbon widths while finite gap opening has been observed in certain widths of armchair silicene nanoribbons (ASiNRs) [6]. 


\section{MODELLING IMPLEMENTATION}

For the ease of description, nanoribbon of width, $N_{z}$ is denoted as $N_{z}$-ZSiNR where $N_{z}$ is defined as the number of zigzag chains across the $N_{z}$-ZSiNR. Furthermore, the length of $N_{z}$-ZSiNR is denoted by $L$ which represents the number of unit cells along the nanoribbon length direction. Both $N_{z}$ and $L$ are positive integers. Figure 2 depicts the structural model of defective ZSiNR. In this work, we focus on 4-ZSiNR with $L=2$ while varying the SV defect locations (A, B, C and D). The structure in Figure 2 is assumed to be perfect planar hexagonal and stable despite the SV defect. The following subsections discuss the mathematical models to compute band structures and density of states (DOS).

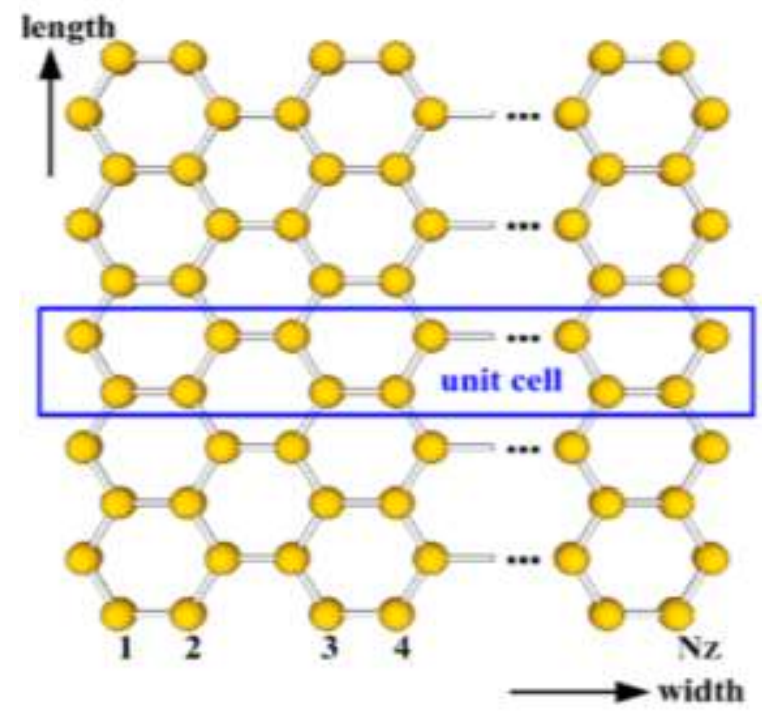

Figure 1. The schematic diagram for the atomic structure of ZSiNR [14]. The length, is defined as the number of unit cell in the SiNR and the width is denoted as $N_{z}$

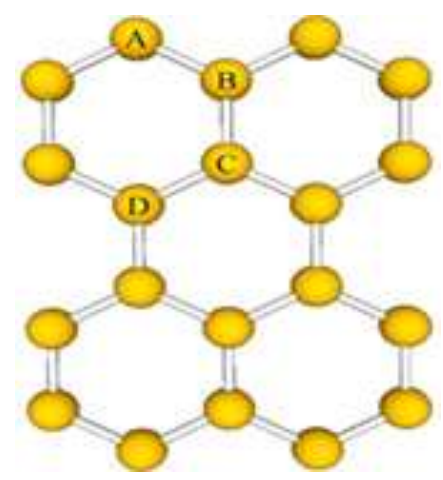

Figure 2. The schematic diagram for the SV defect locations (A, B, C and D) for 4-ZSiNR with $=2$

\subsection{Band structures}

The band structures of the defective ZSiNRs are plotted by using the dispersion relation from the solutions of energy eigenvalues from the Hamiltonian matrices. The defective ZSiNRs are modelled using the NNTB approach based on the time-independent Schrödinger in matrix form [15-17]:

$$
E\left\{\phi_{0}\right\}=[h(\vec{k})]\left\{\phi_{0}\right\},
$$

where $h(\vec{k})$ is the Hamiltonian matrix, 0 is the time-independent wave function and is the energy. In this work, the Hamiltonian matrix is divided into the -matrix and -matrix which describes the interactions of silicon atoms in the zigzag unit cell chain and the interations among the zigzag unit cell chains respectively. Therefore, the complex model is now reduced to a form similar to one-dimensional (1D) problem which can be solved using the simple, given by $[18,19]$ :

$$
h(k)=\beta^{\prime} e^{-i k a}+\alpha+\beta e^{+i k a},
$$

where $\beta^{\prime}$ is the transverse matrix of $\beta$-matrix, $k$ is the wave vector and $a=0.382 \mathrm{~nm}$ [20] is the lattice constant of silicene, but the lattice constant is negligible in this work because the $\mathrm{x}$-axis of the band structure is $\mathrm{ka} / \pi$.

The bonding energy, $t$ used in this study is $-1.03 \mathrm{eV}$ adapted from previous study [21]. The standard eigenvalue problem solution, $\operatorname{det}|[h(k)]-\varepsilon(k) I|=0$ is employed to evaluate the $h(k)$ to obtain the energy eigenvalues, where $I$ is an identity matrix of the same size as $h(k)$ and $\varepsilon(k)$ is the energy eigenstates. 


\subsection{Density of states}

DOS determines the number of available states in a semiconductor material for the carriers to flow, which is essential to obtain the current flow in the system [22]. The numerical solution of DOS for a system can be obtained using delta, $\delta$ function [13], given as:

$$
\operatorname{DOS}(E)=\sum_{i=1}^{N} \int_{-\infty}^{+\infty} \delta[E-\varepsilon(k)] d k .
$$

where $\varepsilon(k)$ is the energy eigenstates as described in previous subsection. By applying the spectral function identity [23] as shown in (4) and the integration rule for $\delta$ function, the numerical solution for DOS as a function of energy, $E$ can be simplified as (5). The are given as:

$$
\begin{gathered}
\delta[E-\varepsilon(k)]=\frac{1}{2 \pi}\left[\frac{2 \eta}{[E-\varepsilon(k)]^{2}+\eta^{2}}\right]_{\eta \rightarrow 0^{+}}, \\
\operatorname{DOS}(\mathrm{E})-\sum_{i=1}^{N} \frac{1}{2 \pi} \sum_{\text {all } k} \frac{2 \eta}{[E-\varepsilon(k)]^{2}+\eta^{2}},
\end{gathered}
$$

where $\eta$ is a very small positive value to prevent the inverse matrix from diverging. The DOS is calculated by iterating $E$ from the minimum to the maximum value of $(k)$.

\section{RESULT AND DISCUSSION}

In this section, the NNTB model is benchmarked with a more sophisticated computational study (first-principle study) to ensure the parameters are accurate. Then, the results for the pristine and defected 4-ZSiNRs are shown and discussed. The band structures and DOS of pristine and defected 4-ZSiNRs are plotted in the same graph to examine the effects of SV defect to the electronic properties.

\subsection{Benchmark of pristine zigzag silicene nanoribbons}

The parameters of NNTB model is benchmarked with the same structure of 6-ZSiNR with $L=1$ from Figure 3(a) in [6]. This step is done to ensure the accuracy of the NNTB model. Figure 3 depicts the comparison between the proposed model and published result. The graph in Figure 3(a) shows that NNTB is consistent with the more computationally expensive calculations.

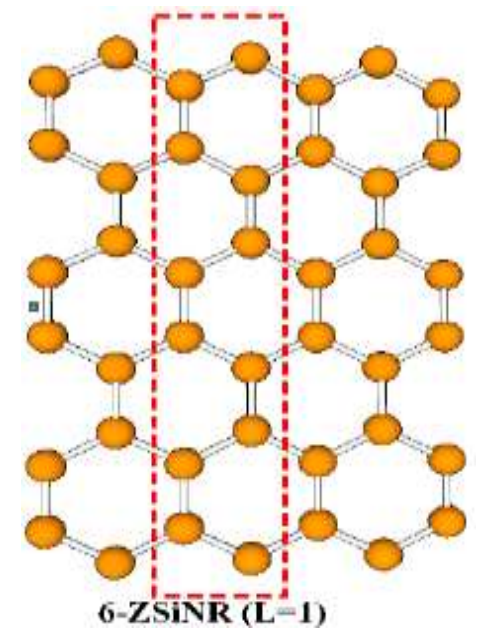

(a)

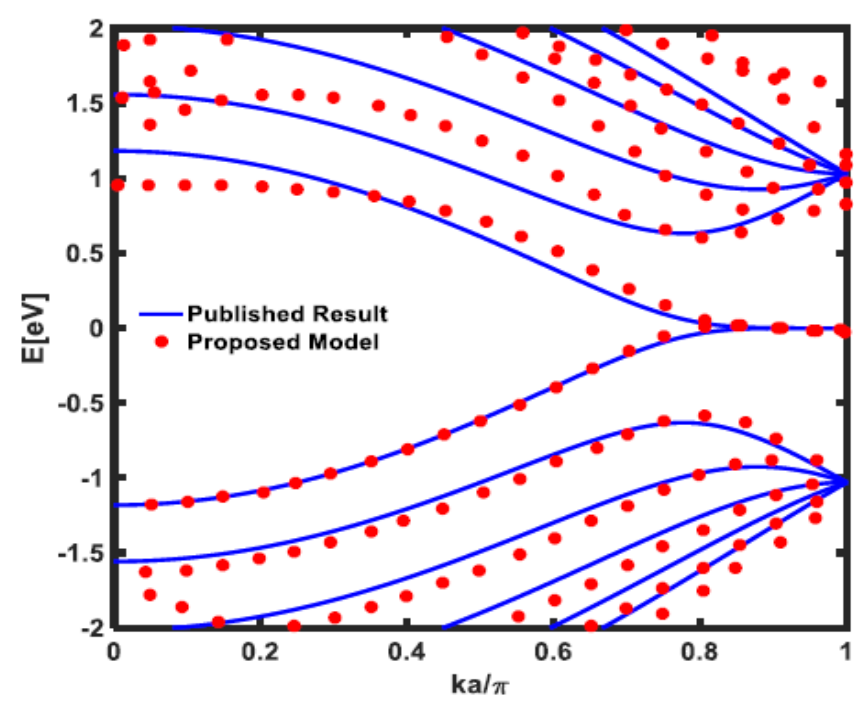

(b)

Figure 3. The benchmark of band structure for pristine $6-Z S i N R$ with $L=1$. Blue solid lines show the band structure of present work and red dots show the band structure adapted from first-principle calculations (a) Lattice Structure, (b) Band Structure [6] 


\subsection{Pristine zigzag silicene nanoribbons}

Figure 4 depicts the band structures and DOS for pristine 4-ZSiNR with $L=2$.The band structure shows that the valence band maximum $(\mathrm{VBM})$ and the conduction band minimum $(\mathrm{CBM})$ touches at $E=0 \mathrm{eV}$ and the extracted band gap is zero by calculating the difference between VBM and CBM.
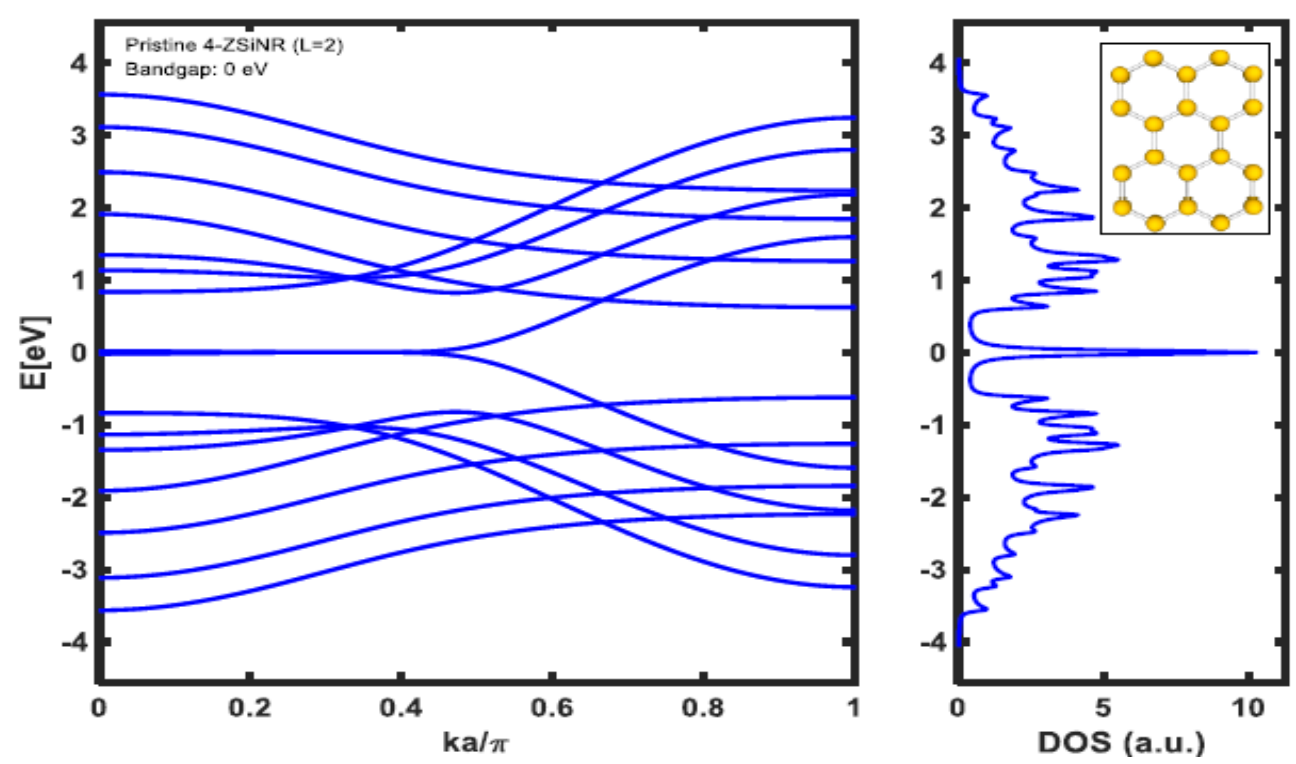

Figure 4. The band structure and DOS for pristine 4-ZSiNR with $L=2$

\subsection{Defective zigzag silicene nanoribbons}

Figures 5(a) to (d) depict the band structures and DOS for defective 4-ZSiNR with $L=2$ for SV locations of A, B, C and D respectively. For all the simulated SV locations, the extracted band gaps are zero. Moreover, all the SV locations affect shapes of the band structures and DOS when compared to the band structures and DOS of the pristine 4-ZSiNR. Figures 5(a) and (b) show that the CBM and VBM touches at $E=0 \mathrm{eV}$ and Figures 5(c) and (d) show that the CBM and VBM overlaps at $E=0 \mathrm{eV}$ (or in other words, the conduction band is below Fermi level and valence band is above Fermi level).

\subsection{Discussions}

By comparing Figure 3 to Figures 5(a) to (d), it is shown that the SV defect does not have significant effects to the bandgaps for $4-Z S i N R$ with $L=2$ for all the simulated SV defect locations (A, B, C and D). Similar results were also shown by previous study on zigzag graphene nanoribbons (ZGNRs) where the study had concluded that SV defect does not bring significant effect to the bandgaps of ZGNRs [24]. On the other hand, there are interesting points to notice from the band structures. For SV locations at $\mathrm{A}$ and $\mathrm{B}$, the defected 4-ZSiNRs show semi-metallic properties. In addition, the overlapping of conduction and valence bands for SV locations at $\mathrm{C}$ and $\mathrm{D}$ indicates metallic properties [25].

The shapes of the band structures are distorted in different ways when the SV defect locations are varied. The peaks of DOS at $E=0 \mathrm{eV}$ for defected 4-ZSiNR increase significantly compared to pristine 4-ZSiNR. The peaks in the DOS are crucial for transport properties which can affect the current versus voltage characteristics of electronic devices [26]. The DOS is a quantity of the material that indicates the number of available states for conduction [27]. Higher peaks of DOS at $E=0 \mathrm{eV}$ for defected 4-ZSiNRs imply that there is more number of available states for carriers to flow around the Fermi energy level compared to pristine 4-ZSiNR. 


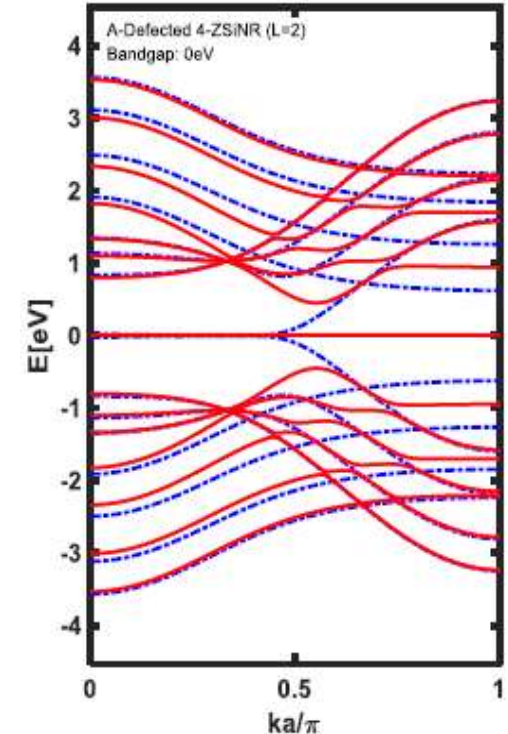

(a)

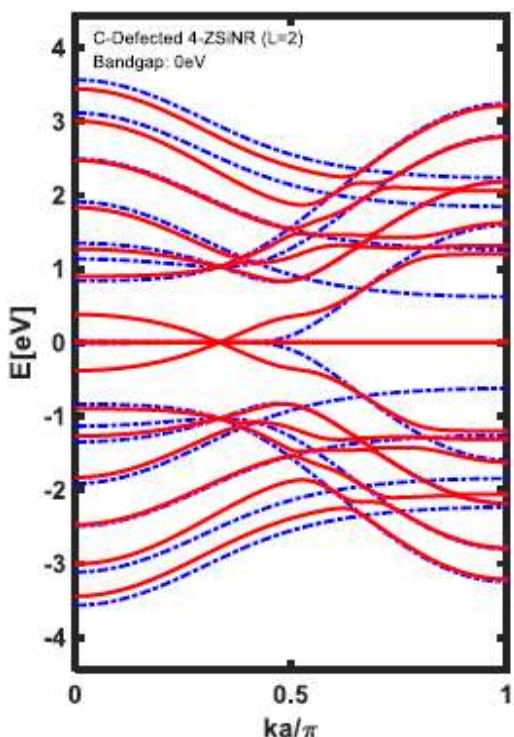

(c)
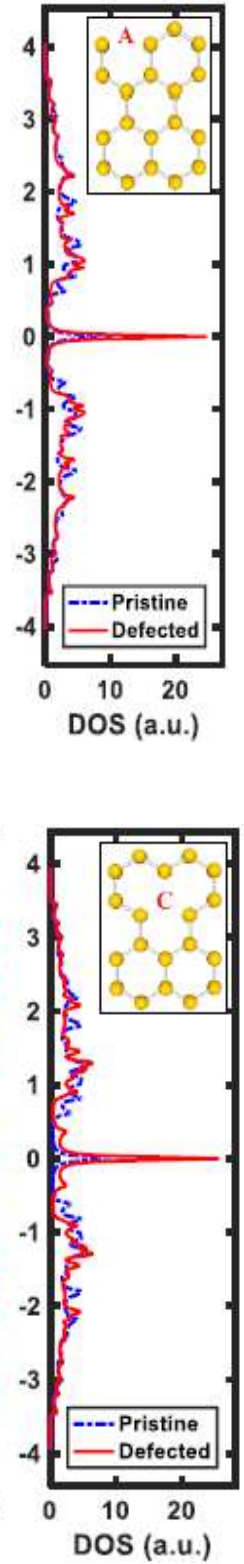

DOS (a.u.)
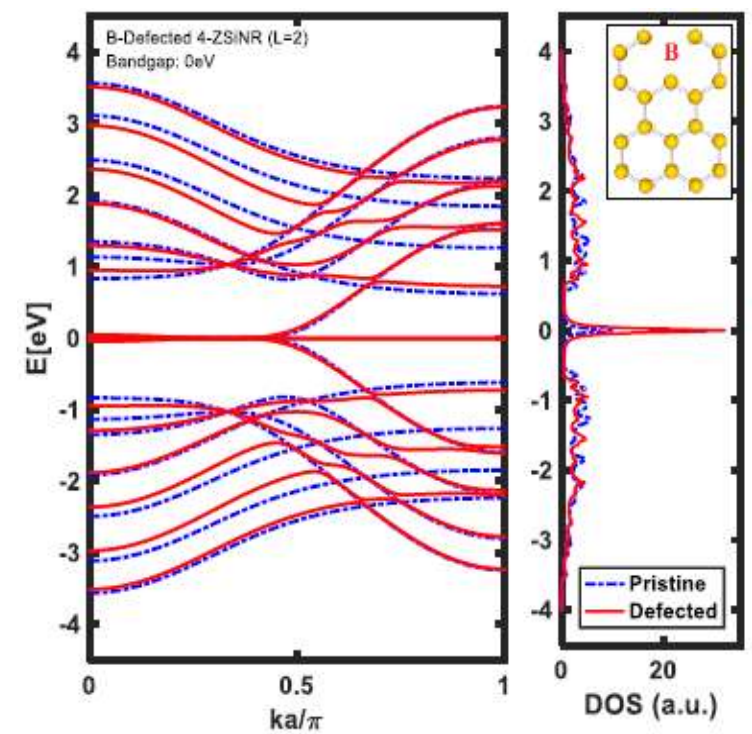

(b)
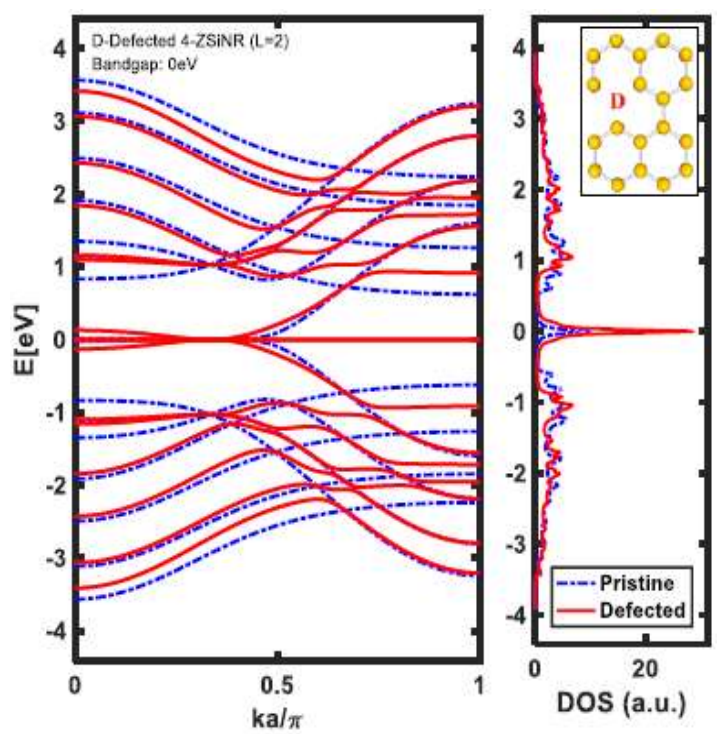

(d)

Figure 5. The band structures and DOS for defected 4-ZSiNR with $L=2$ for various SV locations. Blue dotted lines represent the results for pristine nanoribbons and red solid lines represent the results for defected nanoribbons. The schematic diagram for each defected 4-ZSiNR is shown at the top right corner of each result, (a) Defected (SV Location=A), (b) Defected (SV Location=B), (c) (Defected (SV Location=C), (d) Defected (SV Location=D)

\section{CONCLUSION}

The electronic properties, namely band structures and density of states of pristine and defective ZSiNRs of various lengths have been modelled and simulated using NNTB. The results show that SV defect does not have significant impact on the bandgaps of 4-ZSiNR with $L=2$ when the locations of SV defects are varied. However, the SV defect can shift and distort the band structures and increase the peak of DOS. This work can be extended by investigating the electronic properties for ZSiNR by varying the vacancy concentrations or widths and lengths of nanoribbons. 


\section{ACKNOWLEDGEMENTS}

The authors acknowledge the Research Management Centre (RMC) of Universiti Teknologi Malaysia (UTM) for providing excellent support and a stimulating research environment. Mu Wen would like to convey his gratitude for the award of PhD Zamalah Scholarship from the School of Graduate Studies, UTM. Michael Tan would like to acknowledge the financial support from UTM Fundamental Research (UTMFR) (Vote no.: Q.J130000.2551.21H51), which allowed the smooth progress of this research.

\section{REFRENCES}

[1] K. S. Novoselov et al., "Electric field effect in atomically thin carbon films," Science, vol. 306, no. 5696, pp. 666-669, 2004. https://doi.org/10.1126/science.1102896

[2] G. R. Bhimanapati et al., "Recent advances in two-dimensional materials beyond graphene," ACS nano, vol. 9, no. 12, pp. 11509-11539, 2015. https://doi.org/10.1021/acsnano.5b05556

[3] C. L. Ng and M. L. P. Tan, "Low dimensional simulator for carbon-based devices," in 2014 IEEE International Conference on Semiconductor Electronics (ICSE2014), pp. 40-43, 2014.

[4] M. W. Chuan et al., "2D Honeycomb Silicon: A Review on Theoretical Advances for Silicene Field-Effect Transistors," Current Nanoscience, 2020. https://doi.org/10.2174/1573413715666190709120019

[5] J. Zhao et al., "Rise of silicene: A competitive 2D material," Progress in Materials Science, vol. 83, pp. 24-151, 2016. https://doi.org/10.1016/j.pmatsci.2016.04.001

[6] Y. Ding and J. Ni, "Electronic structures of silicon nanoribbons," Applied Physics Letters, vol. 95, no. 8, pp. 083115, 2009. https://doi.org/10.1063/1.3211968

[7] G. G. Guzmán-Verri and L. L. Y. Voon, "Electronic structure of silicon-based nanostructures," Physical Review B, vol. 76, no. 7, pp. 075131, 2007. https://doi.org/10.1103/PhysRevB.76.075131

[8] K. Takeda and K. Shiraishi, "Theoretical possibility of stage corrugation in $\mathrm{Si}$ and Ge analogs of graphite," Physical Review B, vol. 50, no. 20, pp. 14916, 1994. https://doi.org/10.1103/physrevb.50.14916

[9] H. Dong, D. Fang, B. Gong, Y. Zhang, E. Zhang, and S. Zhang, "Electronic and magnetic properties of zigzag silicene nanoribbons with Stone-Wales defects," Journal of Applied Physics, vol. 117, no. 6, pp. 064307, 2015. https://doi.org/10.1063/1.4907582

[10] L. Tao et al., "Silicene field-effect transistors operating at room temperature," Nature nanotechnology, vol. 10, no. 3, pp. 227, 2015. https://doi.org/10.1038/nnano.2014.325

[11] S. Li et al., "Defects in silicene: vacancy clusters, extended line defects, and di-adatoms," Scientific reports, vol. 5, pp. 7881, 2015. https://doi.org/10.1038/srep07881

[12] K. L. Wong, M. W. Chuan, N. E. Alias, A. Hamzah, C. S. Lim, and M. L. P. Tan, "Modeling of low-dimensional pristine and vacancy incorporated graphene nanoribbons using tight binding model and their electronic structures," Advances in nano research, vol. 7, no. 3, pp. 207-219, 2019. https://doi.org/10.12989/anr.2019.7.3.209

[13] H. Terrones, R. Lv, M. Terrones, and M. S. Dresselhaus, "The role of defects and doping in 2D graphene sheets and 1D nanoribbons," Reports on Progress in Physics, vol. 75, no. 6, pp. 062501, 2012. https://doi.org/10.1088/00344885/75/6/062501

[14] L. Lew Yan Voon, J. Zhu, and U. Schwingenschlögl, "Silicene: Recent theoretical advances," Applied Physics Reviews, vol. 3, no. 4, pp. 040802, 2016. https://doi.org/10.1063/1.4944631

[15] W. H. Lim, A. Hamzah, M. T. Ahmadi, and R. Ismail, "Analytical study of the electronic properties of boron nitride nanosheet," in 2017 IEEE Regional Symposium on Micro and Nanoelectronics (RSM), pp. 42-45, 2017. https://doi.org/10.1109/RSM.2017.8069115

[16] S. Datta, "Lessons from nanoelectronics: a new perspective on transport," World Scientific publishing company, 2012. https://doi.org/10.1142/8029

[17] M. W. Chuan et al., "Electronic properties and carrier transport properties of low-dimensional aluminium doped silicene nanostructure," Physica E: Low-dimensional Systems and Nanostructures, vol. 116, pp. 113731, 2020. https://doi.org/10.1016/j.physe.2019.113731

[18] E. Goh, H. C. Chin, K. L. Wong, I. S. B. Indra, and M. L. P. Tan, "Modeling and Simulation of the Electronic Properties in Graphene Nanoribbons of Varying Widths and Lengths Using Tight-Binding Hamiltonian," Journal of Nanoelectronics and Optoelectronics, vol. 13, no. 2, pp. 289-300, 2018. https://doi.org/10.1166/jno.2018.2206

[19] K. Wakabayashi, Y. Takane, M. Yamamoto, and M. Sigrist, "Electronic transport properties of graphene nanoribbons," New Journal of Physics, vol. 11, no. 9, pp. 095016, 2009. https://doi.org/10.1088/13672630/11/9/095016

[20] L. Lew Yan Voon, E. Sandberg, R. Aga, and A. Farajian, "Hydrogen compounds of group-IV nanosheets," Applied Physics Letters, vol. 97, no. 16, pp. 163114, 2010. https://doi.org/10.1063/1.3495786

[21] N. J. Roome and J. D. Carey, "Beyond graphene: stable elemental monolayers of silicene and germanene," ACS applied materials \& interfaces, vol. 6, no. 10, pp. 7743-7750, 2014. https://doi.org/10.1021/am501022x

[22] M. S. Lundstrom and D. A. Antoniadis, "Compact models and the physics of nanoscale FETs," IEEE Transactions on Electron Devices, vol. 61, no. 2, pp. 225-233, 2014. https://doi.org/10.1109/TED.2013.2283253

[23] S. Datta, "Quantum transport: atom to transistor," Cambridge university press, 2005. https://doi.org/10.1017/CBO9781139164313

[24] K. L. Wong et al., "Influence of single vacancy defect at varying length on electronic properties of zigzag graphene nanoribbons," Indonesian Journal of Electrical Engineering and Informatics (IJEEI), vol. 7, no. 2, pp. 366-374, 2019. https://doi.org/10.11591/ijeei.v7i2.1138 
[25] D. A. Neamen, "Semiconductor physics and devices: basic principles". New York, NY: McGraw-Hill, 2012.

[26] W. H. Lim, A. Hamzah, M. T. Ahmadi, and R. Ismail, "Performance analysis of one dimensional BC2N for nanoelectronics applications," Physica E: Low-dimensional Systems and Nanostructures, vol. 102, pp. 33-38, 2018. https://doi.org/10.1016/j.physe.2018.04.005

[27] V. K. Arora, "Nanoelectronics: Quantum engineering of low-dimensional nanoensembles," CRC Press, 2015. https://doi.org/10.1201/9781315222516

\section{BIOGRAPHIES OF AUTHORS}
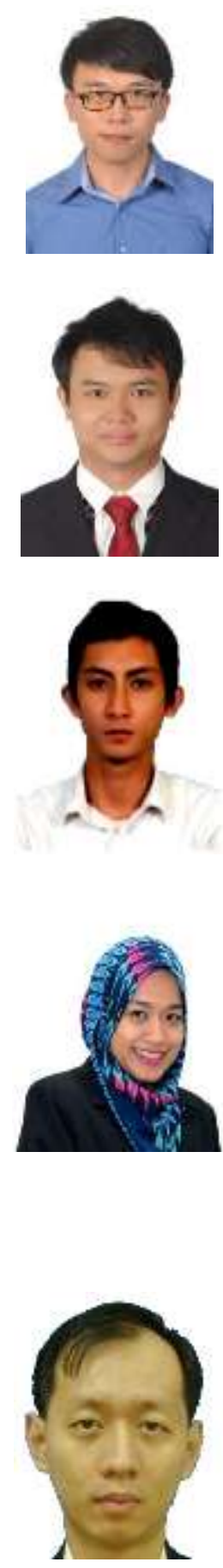

MU WEN CHUAN was born in Melaka, Malaysia in 1994. He received B.Eng. (electrical-electronics) degree from Universiti Teknologi Malaysia, in 2018. He is currently pursuing the $\mathrm{PhD}$ degree in electrical engineering at UTM. His research interests are in device modelling and circuit simulation of MOSFET devices.

KIEN LIONG WONG was born in Skudai, Johor, Malaysia in 1993. He received the B.Eng. (electrical-electronics) degree from Universiti Teknologi Malaysia in 2017 and is currently prsuing the PhD degree in Electrical Engineering at UTM. He is working on the tightbinding quantum transport in graphene nanoribbons (GNRs) with line edge roughness and doping. His research interests are in device modelling of carbon-based material and fieldeffect transistor (FET) devices.

AFIQ HAMZAH received his B.Eng. (2011), M.Eng. (2014) and PhD (2018) in Electrical and Electronics Engineering from the Universiti Teknologi Malaysia. He is currently a senior lecturer with the School of Electrical Engineering, Universiti Teknologi Malaysia. His research interest includes virtual fabrication process, device compact modeling of multiple-gate FETs and graphene-based devices, and circuit simulation

NURUL EZAILA ALIAS received her B.Eng. in electrical and electronics engineering from Universiti Teknologi MARA, Malaysia (2007), M.Eng. in Electronics and Telecommunication Engineering from Universiti Teknologi Malaysia (2010). She then received her $\mathrm{PhD}$ degree in Electrical Engineering and Information System from The University of Tokyo (2014). She is currently a senior lecturer with thr Division of Electronic and Computer Engineering at UTM. She is a member of IEEE Society Malaysia Section, IEEE Electron Devices Society and The Institution of Engineers Malaysia (IEM). She has reviewed several international journals namely IEEE Transactions on Very Large-Scale Integration (VLSI) Systems. She is also active in supervising local and international postgraduate students. Her research interests is in advanced TCAD simulations and analysis of semiconductor devices, design techniques of FET-based memory application, variability and reliability issue in nanoscale FET devices.

CHENG SIONG LIM received his B.Eng. degree in Electrical Engineering (First Class Honors), M.Eng. degree (Electrical), and $\mathrm{PhD}$ degree from Universiti Teknologi Malaysia (UTM), in 1999, 2004, and 2011, respectively. He is a senior lecturer in the School of Electrical Engineering, Faculty of Engineering, UTM. His research interests are in embedded system, emergency medical services, telerobotics and multi-agent system. 


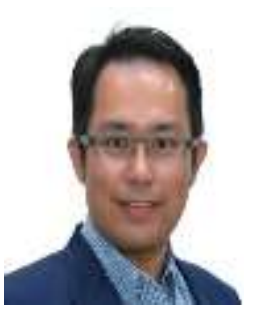

MICHAEL LOONG PENG TAN was born in Bukit Mertajam, Penang, Malaysia, in 1981. He obtained his Bachelor of Engineering and was appointed as a tutor in the same university in September 2003. He started his Master by research on June 2004 and carried out his research as a graduate intern in Penang Design Centre (PDC) Intel Technology Sdn. Bhd, Penang. He was awarded Masters of Engineering from UTM in 2006. Subsequently, Michael pursued his $\mathrm{PhD}$ in Engineering at Queens' College, Cambridge from October 2007 until June 2011. He was awarded his PhD degree in July 2011 at the University of Cambridge, United Kingdom. Presently, he is a senior lecturer and researcher in the field of low dimensional nanoelectronics devices. He is the head of Computational Nanoelectronics (CoNE) Research Group. In addition, he is a Chartered Engineer registered under the Engineering Council, United Kingdom. He is a Professional Engineer with Practising Certificate (PEPC) with the Board of Engineers Malaysia (BEM) and a lifetime corporate member of the Institution of Engineers Malaysia (IEM). He is also member of the Institution of Engineering and Technology (IET) and Institute of Physics (IOP), life member of the American Physical Society (APS) and senior member of IEEE (SMIEEE). His research interests are in device modeling and circuit simulation of silicon and carbon-based nanotransistor. 